\title{
Population-based description of familial clustering of Chiari malformation Type I
}

\author{
Diana Abbott, PhD, ${ }^{1}$ Douglas Brockmeyer, MD, ${ }^{2}$ Deborah W. Neklason, PhD, ${ }^{1}$ Craig Teerlink, PhD, ${ }^{1}$ \\ and Lisa A. Cannon-Albright, $\mathrm{PhD}^{1,3}$
}

\begin{abstract}
'Division of Genetic Epidemiology, Department of Internal Medicine, and 2Department of Neurosurgery, Clinical Neurosciences Center, University of Utah School of Medicine; and ${ }^{3}$ George E. Wahlen Department of Veterans Affairs Medical Center, Salt Lake City, Utah
\end{abstract}

OBJECTIVE A population-based genealogical resource with linked medical data was used to define the observed famil-
ial clustering of Chiari malformation Type I (CM-I).
METHODS All patients with CM-I were identified from the 2 largest health care providers in Utah; those patients with
linked genealogical data were used to test hypotheses regarding familial clustering. Relative risks (RRs) in first-, second-,
and third-degree relatives were estimated using internal cohort-specific CM-I rates; the Genealogical Index of Familial-
ity (GIF) test was used to test for an excess of relationships between all patients with CM-I compared with the expected
distribution of relationships for matched control sets randomly selected from the resource. Pedigrees with significantly
more patients with CM-I than expected $(p<0.05)$ based on internal rates were identified.

RESULTS A total of 2871 patients with CM-I with at least 3 generations of genealogical data were identified. Significantly increased RRs were observed for first- and third-degree relatives (RR 4.54, $p<0.001$, and RR 1.36, $p<0.001$, respectively); the RR for second-degree relatives was elevated, but not significantly (RR 1.20, $p=0.13)$. Significant excess pairwise relatedness was observed among the patients with $\mathrm{CM}-\mathrm{I}(\mathrm{p}<0.001)$, and borderline significant excess pairwise relatedness was observed when all relationships closer than first cousins were ignored $(p=0.051)$. Multiple extended high-risk CM-I pedigrees with closely and distantly related members were identified.

CONCLUSIONS This population-based description of the familial clustering of 2871 patients with CM-I provided strong evidence for a genetic contribution to a predisposition to CM-I.

https://thejns.org/doi/abs/10.3171/2016.9.JNS161274

KEY WORDS Chiari malformation; relative risk; UPDB; familiality; skull base

$\mathrm{C}$ HIARI malformation Type I (CM-I) is a neurological and spinal disorder characterized by herniation of The underlying cause of the disorder is unknown. CM-I frequently presents with other significant radiographic findings, including caudal migration of the brainstem, odontoid retroflexion, basilar invagination, occipitalization of the atlas, syringomyelia, and scoliosis, all observed either alone or in combination with simple tonsillar herniation. CM-I may cause a wide variety of signs and symptoms, most of which are believed to be the result of abnormalities in CSF flow and/or brainstem compression.

Estimates suggest that approximately 215,000 individu- als in the US may be affected with CM-I, ${ }^{18}$ and approximately $12 \%$ of these patients have at least 1 close relative with the disorder. ${ }^{14}$ In a population-based retrospective cohort study, Aitken et al. ${ }^{1}$ demonstrated that the prevalence of CM-I in 5248 asymptomatic and symptomatic patients under 20 years of age was $0.97 \%$, thus underlining the frequency of the disorder. However, the true prevalence of CM-I is difficult to determine because of several factors, most notably the presence of asymptomatic individuals within the population, the discovery of incidental findings on imaging, and a "detection effect" in which patients might undergo MRI screening because a family member carries the CM-I diagnosis. As a result of these confound-

ABBREVIATIONS CI = confidence interval; $\mathrm{CM}-\mathrm{I}$ = Chiari malformation Type I; $\mathrm{dGIF}=$ distant GIF; GIF = Genealogical Index of Familiality; RR = relative risk; UPDB = Utah Population Database.

SUBMITTED May 19, 2016. ACCEPTED September 28, 2016.

INCLUDE WHEN CITING Published online February 3, 2017; DOI: 10.3171/2016.9.JNS161274. 
ing factors, establishing a clear picture of the imaging indications in large data sets can be difficult.

Many other studies of CM-I have reported familial clustering, co-occurrence in twins, and an overlap with a subset of known genetic syndromes, ${ }^{2,12,14,16,19,20}$ suggesting a strong genetic predisposition to this disorder. However, the heritable basis of CM-I has never been investigated using a state-wide population-based data set with sophisticated epidemiological techniques. The information gained from such an analysis would inform conversations with patients and families about the inherited risk of CM-I and strengthen our understanding of the genetic basis of the disorder.

The analysis reported here uses the Utah Population Database (UPDB), a genealogy database linked to statewide medical data, to define the observed familial clustering for the CM-I phenotype using a large cohort of patients with CM-I identified from the 2 major health care providers for the state of Utah.

\section{Methods \\ UPDB Genealogy}

The UPDB is a population-based resource linking Utah health and hospital record information to a computerized genealogy of the original Utah pioneers and their descendants. The initial genealogy data for 1.6 million individuals was provided by the Church of Jesus Christ of LatterDay Saints Family History Library in the 1970s. ${ }^{17}$ Over the years, the genealogy has expanded via regular updates from Utah state vital records (e.g., father, mother, and child from birth certificates) to include a large part of the Utah population. The UPDB now includes over 2.8 million individuals with at least 3 generations of genealogical data that connect to the original genealogy data. Multiple Utah data repositories have been linked to the Utah genealogy data in the UPDB, including the electronic data warehouses for the 2 largest health care providers in Utah, which cover $60 \%-70 \%$ of Utah individuals. After we obtained institutional review board approval, clinical data for this study were obtained from the University of Utah Health Sciences Center and Intermountain Healthcare. Data from 1994 for these providers was independently record-linked to the UPDB genealogy; 767,594 linked patients from the University of Utah Health Sciences Center and 1,750,935 linked patients from Intermountain Healthcare have at least 3 generations of genealogical data.

\section{Clinical Classification}

The ICD-9 diagnosis code 348.4 for compression of brain and/or the CPT code 61343 for Chiari decompression were used to identify patients.

\section{Estimation of Relative Risk for CM-I in Relatives}

Estimation of relative risks (RRs) in relatives is a common method to test for evidence of a genetic contribution for a phenotype. RRs were estimated using all patients identified in either of the 2 health care systems. The observed number of patients with CM-I for a group of rela- tives was obtained by counting (without duplication) the number of relatives diagnosed with CM-I across all cohorts. The expected number of affected relatives was estimated using cohort-specific population disease rates for CM-I estimated from the UPDB. Each individual in the UPDB with genealogical data was assigned to 1 of 136 cohorts based on sex, birth state (Utah or outside of Utah), and birth year (5-year cohorts). The cohort-specific disease rate for CM-I was estimated as the number of patients with CM-I in each cohort divided by the total number of UPDB individuals in the cohort. The expected number of patients with CM-I for a group of relatives was estimated by multiplying the total number of relatives in each cohort by the cohort-specific CM-I rate, then summing across all cohorts. The ratio of observed to expected number of affected relatives is an unbiased estimator of the RR. Significantly elevated RRs in first-degree relatives may represent either shared genetics or shared environment; significantly elevated RRs in more distant relatives, however, strongly suggests a genetic contribution to risk, rather than simply shared environment. RRs were estimated independently for first-, second-, and third-degree relatives. First-degree relatives are parents, offspring, and siblings; second-degree relatives are the first-degree relatives of all first-degree relatives (e.g., father's brother, or uncle); third-degree relatives are the first-degree relatives of all second-degree relatives (e.g., father's brother's daughter, or cousin).

\section{Genealogical Index of Familiality}

The Genealogical Index of Familiality (GIF) method was used to test for excess relatedness in individuals with CM-I. This method was developed to study familial aggregation of disease within a genealogy, and it involves an observed/expected comparison of familial clustering using the coefficient of kinship. The coefficient of kinship is a measure of relatedness commonly used to define pairs of individuals; it represents the probability that 2 alleles, sampled at random from each individual, are identical by descent from a common ancestor. By using the coefficient of kinship, ${ }^{8}$ the relatedness was calculated for all possible pairs of patients with CM-I. For most pairs of individuals considered, no relationship was observed. For the GIF test, the average relatedness measure for all of the pairs of patients with CM-I was compared with the expected average relatedness estimated from 1000 matched-control sets randomly selected from the UPDB. Each control set was formed by randomly selecting a cohort-matched control for each patient with CM-I. The empirical significance of the GIF test for excess relatedness is based on comparison of the mean pairwise relatedness of the patients with CM-I to the distribution of the mean pairwise relatedness for the 1000 matched-control sets.

The GIF statistic analysis for CM-I tested for an excess of pairwise relatedness of the patients with CM-I over the expected relatedness of a group of similar individuals in the UPDB population. Significant excess relatedness (or familial clustering) could be due to shared environment, shared genes, or both. To minimize the effects of shared environment on familial clustering, an additional test, the distant GIF (dGIF), was also performed. The dGIF statistic is calculated in the same manner as the GIF, except that 


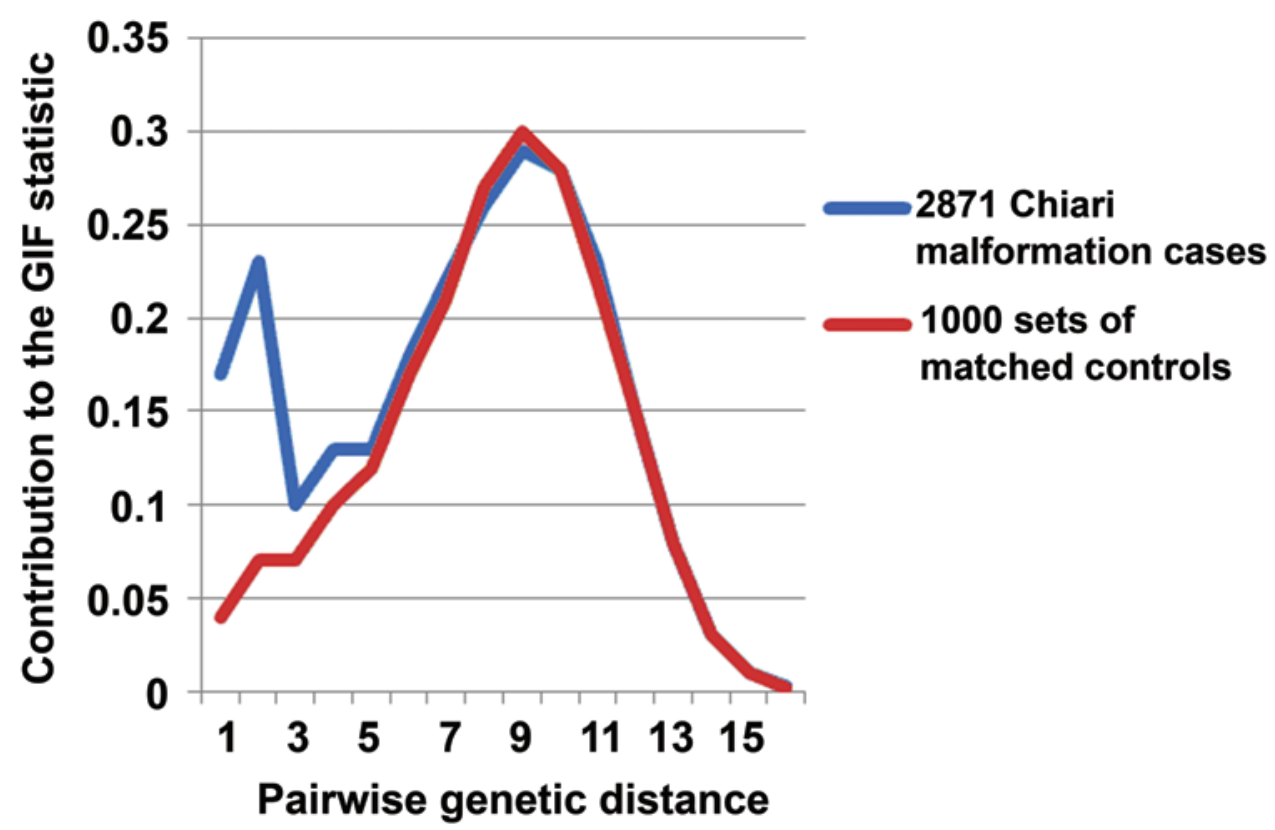

FIG. 1. Contribution to the GIF statistic by pairwise genetic distance for 2871 patients with CM-I compared with matched controls. Pairwise genetic distance: 1 = parent/offspring; 2 = siblings or grandparent/grandchild; 3 = avuncular relatives, for example; 4 = first cousins, for example; 5 = first cousins, once removed; 6 = second cousins, and so forth. Figure is available in color online only.

it ignores all relationships closer than first cousins. The dGIF statistic therefore tests for excess relatedness when only distant relationships are considered.

The contribution to the GIF statistic from each different type of relationship (close and distant) can be displayed by the pairwise genetic distance for all pairs of study patients and controls, allowing consideration of which relationships contributed most to any observed excess relatedness (Fig. 1). The pairwise genetic distances (x-axis) represent parent/offspring (1), siblings or grandparent/grandchild (2), uncle/niece or similar avuncular (3), first cousins or similar (4), second cousins (6), and so forth, and the contribution to the overall GIF statistic can be seen by relationship. There are more pairs of relationships of each type as the genetic distance of the pair increases, but the coefficient of kinship is smaller for more distant relationships (e.g., 0.25 for siblings, 0.0625 for first cousins, and so forth). In Fig. 1, the distribution shown for the mean GIF for the 1000 sets of matched controls shows the expected distribution of relationships of all pairs in a group of individuals from Utah who are similar to the study patients with CM-I (matched for sex, 5-year birth year range, and birth state [Utah or not]); this distribution is very similar to the control distribution observed for most studies and represents the typical relatedness structure for a random group of individuals selected from the UPDB.

\section{High-Risk Pedigrees}

High-risk CM-I pedigrees, defined as pedigrees that have a statistically significant excess of pedigree members with CM-I, were identified. All related clusters of patients with CM-I descending from a common founder pair were identified in the UPDB. Because these clusters of related patients (or pedigrees) may have occurred by chance, a test for a significant excess of patients with CM-I in each pedigree was performed. Using cohort-specific CM-I rates applied to the descendants, the expected number of persons with CM-I was estimated for each pedigree. The ratio of the observed to the expected number of persons with CM-I was calculated and used to assess whether there was a significant excess of individuals with CM-I in the pedigree. Pedigrees with a significant excess of individuals with CM-I $(\mathrm{p}<0.05)$ were termed high risk.

\section{Results}

A total of 2871 patients diagnosed with CM-I with at least 3 generations of genealogical data were identified from the 2 Utah health care systems; 9 patients had only a CPT procedure code for decompression, 244 patients had both the ICD-9 diagnosis code and the procedure code, and 2618 of the identified patients had only the diagnosis code present at least once in their linked medical record.

RRs for CM-I in first-, second-, and third-degree relatives of patients are shown in Table 1. Significantly increased RRs were observed for first- and third-degree relatives (RR 4.54, p < 0.00001, and RR 1.36, p = 0.000054, respectively). The RR observed in second-degree relatives was elevated, but not significantly (RR 1.20, $p=0.13$ ). Because second-degree relatives are primarily in different generations (for example, grandparent/grandchild or uncle/niece), the narrow window of view on diagnoses (data available from 1994) precludes observation of many such relationships and may explain these results. RRs for the 3 first-degree relative types occurring in different generations (parents, offspring, siblings) were estimated separately but did not differ significantly by type (RR for 3916 parents $=4.23,95 \%$ confidence interval $[\mathrm{CI}] 2.81-6.11$; RR for 7219 siblings $=4.94,95 \%$ CI 3.79-6.31; RR for 4321 children $=4.00,95 \%$ CI 2.66-5.78). Some individu- 
TABLE 1. Estimated relative risks for CM-I in relatives of $2871 \mathrm{CM}-\mathrm{I}$ probands

\begin{tabular}{lcccccc}
\hline \multicolumn{1}{c}{ Relative Type } & No. of Relatives & Observed & Expected & RR & $p$ Value & $95 \% \mathrm{Cl}$ \\
\hline First degree & 15,346 & 119 & 26.2 & 4.54 & $<0.00001$ & $3.76-5.44$ \\
\hline Second degree & 36,010 & 71 & 59.1 & 1.20 & 0.13 & $0.94-1.52$ \\
\hline Third degree & 79,731 & 178 & 130.7 & 1.36 & 0.000054 & $1.17-1.58$ \\
\hline
\end{tabular}

"Expected" refers to the expected number of relatives with a diagnosis of CM-I, whereas "Observed" refers to the observed number of relatives diagnosed with CM-I. First-degree relatives are parents, offspring, and siblings. The first-degree relatives of first-degree relatives are seconddegree relatives, and the first-degree relatives of second-degree relatives are third-degree relatives.

als fit into more than 1 category of first-degree relative of a patient with CM-I. For example, an individual with both an affected parent and an affected sibling would be counted in both categories.

Summary results for the GIF and dGIF tests for excess relatedness are reported in Table 2, which includes the number of patients with CM-I, the mean relatedness of the patients with CM-I (patient GIF), the mean relatedness of the 1000 control sets (mean control GIF), the empirical significance for the GIF test (GIF empirical $p$ value), the mean relatedness of distant relationships for the patients (patient dGIF), the mean relatedness of distant relationships for the 1000 control sets (mean control dGIF), and the empirical $p$ value for the dGIF test (dGIF empirical $p$ value). The overall GIF tests showed significant excess relatedness when all relationships were considered $(\mathrm{p}<$ 0.001 ). When only distant relationships were considered, the dGIF test for excess relatedness was borderline significant $(\mathrm{p}=0.051)$. Figure 1 shows the contribution to the GIF statistic by pairwise genetic distance for patients compared with matched controls; a clear excess of pairwise relationships up to first cousins (genetic distance = 4) was observed for patients compared with controls. We identified 399 high-risk pedigrees $(\mathrm{p}<0.05)$ with between 2 and 79 related patients with CM-I. Figure 2 shows an example of a high-risk Utah CM-I pedigree identified in the UPDB.

\section{Discussion}

This population-based description of the familial clustering of 2871 patients with CM-I observed in the Utah population provides strong evidence for the existence of a genetic contribution to predisposition to CM-I. Significantly elevated RRs were observed in both close and distant relatives. Significant evidence for overall excess relatedness (relationships) was observed, as was borderline-significant evidence for excess relatedness when only distant relationships were considered. Finally, extended pedigrees with a significant excess of patients with CM-I have been identified. This information strengthens our understanding of the familial nature of CM-I. Although familial clustering may occur because of shared environment and shared risk factors, the results presented provide strong evidence that the familial clustering observed for this disease has an inherited contribution.

Previous work has shown a strong tendency for close familial clustering in Chiari malformations. Multiple studies describe a family or families in which more than 1 affected individual is present. ${ }^{4-6,12,15,16,19}$ Inherited disorders may also be associated with CM-I; examples include KlippelFeil syndrome, spondyloepiphyseal dysplasia tarda, Goldenhar syndrome, and achondroplasia, among many others. ${ }^{18}$ This close association with other disorders raises the possibility that both syndromic and nonsyndromic forms of CM-I exist. ${ }^{16}$ Improved ability to identify and observe coaggregation of clinically meaningful phenotypes will be important in answering this question.

By using genomic approaches, other groups have investigated the underlying genetics of CM-I, with preliminary, but promising, results. Boyles et al. ${ }^{3}$ performed genomewide linkage analysis of 71 individuals in 23 families using more than 10,000 single-nucleotide polymorphisms. They found 2-point logarithm of odds (LOD) scores of $>$ 3.0 on chromosomes 15 and 9, implicating the fibrillin-1 gene, which is reported to be important in Marfan syndrome. Markunas et al. ${ }^{11}$ performed genome-wide linkage analysis of 367 individuals in 66 families. They stratified their population into individuals positive or negative for connective tissue disease and found linkage evidence for chromosomes 8 and 12 in connective tissue disease-negative families, implicating the GDF6 and GDF3 genes, both important in Klippel-Feil syndrome.

In a set of 2 publications, Markunas et al.9,10 examined the relationship between cranial base morphometrics and whole-genome expression profiles, finding that much posterior fossa morphology was heritable. Multiple genomic regions were strongly implicated with posterior fossa morphology using ordered subset analysis, including chromosomes 1 and $22 .{ }^{9}$

The analytical methods presented here are well validated; however, this study has some limitations. These include the use of diagnostic or procedure coding in the electronic medical record to identify patients with CM-I.

TABLE 2. GIF test for excess relatedness in 2871 patients with CM-I

\begin{tabular}{ccccccc}
\hline No. w/ CM-I & Patient GIF & Mean Control GIF & GIF Empirical p Value & Patient dGIF & Mean Control dGIF & dGIF Empirical $p$ Value \\
\hline 2871 & 2.51 & 2.12 & $<0.001$ & 2.11 & 2.02 & 0.051 \\
\hline
\end{tabular}

Patient GIF = mean relatedness of the patients; mean control GIF $=$ mean relatedness of the 1000 control sets; patient dGIF = mean relatedness of distant relationships to the patients; mean control dGIF = mean relatedness of distant relationships for the 1000 control sets. 


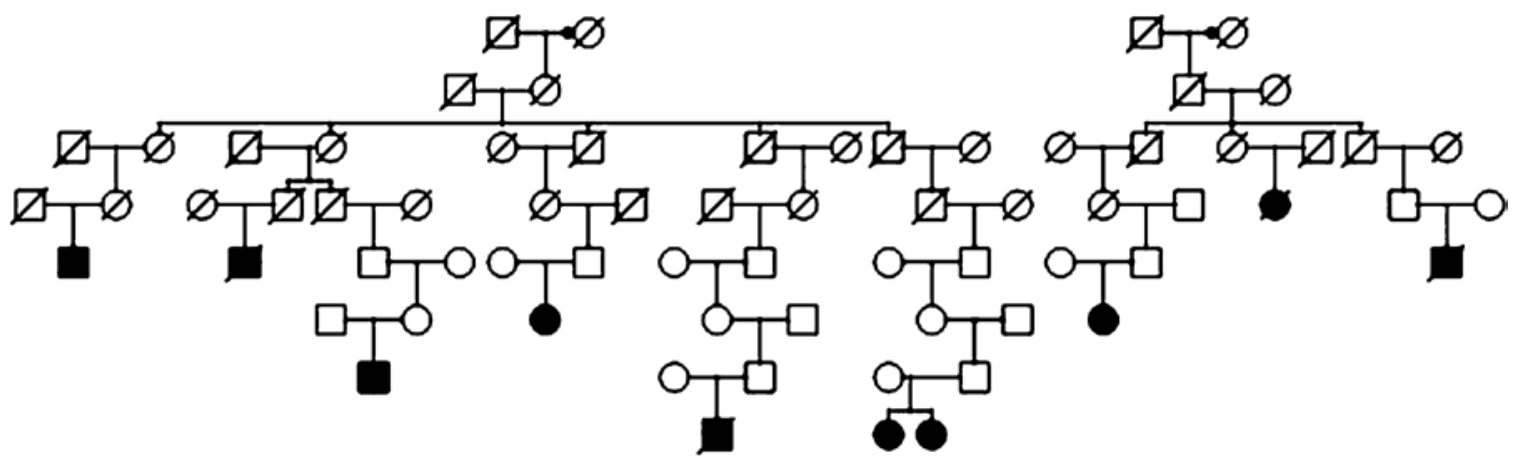

FIG. 2. Example of a high-risk CM-I pedigree. The male pedigree founder has 2 wives and over 6500 descendants in the UPDB, including 10 patients with $\mathrm{CM}-\mathrm{I}$, with only 4.8 patients with $\mathrm{CM}-\mathrm{I}$ expected $(\mathrm{p}=0.02)$. Circles are females, squares are males, strikethrough indicates deceased. The horizontal line between male and female indicates mating (marriage); the line descending from that connects to all their offspring. Multiple marriages have a mark on the mating connector (e.g., the female founder of the pedigree had 2 spouses). Solid shape represents a patient with CM-1.

It is possible that some misclassification of patients may have occurred, but the methods used are very robust to misclassification; missing diagnoses of true instances of CM-I would simply reduce the power of the study and inclusion of false-positive instances of CM-I would similarly reduce the power to identify excess familial relationships, both of which would serve to make all findings conservative in nature.

The study could also be limited by censoring of patients whose linked hospital data did not include appropriate diagnosis or procedure coding. Other potential censoring includes missing or incorrect genealogical data, failure to link matched records, or diagnosis before 1994 or outside the 2 Utah health care systems included. Although there is no ascertainment or referral bias in the study and it is based on medical data for almost the entire Utah population, the possibility of increased frequency of screening (and thus diagnoses) in relatives of patients with CM-I must be recognized. This might affect risks in close relatives, but it is unlikely to explain increased risks in third-degree relatives and extremely unlikely to explain the extended high-risk pedigrees observed.

The Utah population has been shown to be similar to the Northern European populations from which the majority of the Utah pioneers originated. ${ }^{13}$ The results can be assumed to represent similar populations of Northern European extraction but should be validated for other populations.

Given the radiographic and clinical heterogeneity of CM-I, it is no surprise that conclusive evidence of a genetic basis has not been found. Clearly, clues toward a genetic basis of CM-I are beginning to emerge, but much work needs to be done. This population-based analysis of patients with CM-I has provided strong evidence for a genetic contribution to predisposition and evidence of more extended high-risk pedigrees than have previously been reported. This information is important, because it provides a basis for informative discussions with patients and families about the genetic contribution of CM-I. More detailed genomic analysis of the Utah high-risk CM-I pedigrees identified will be important for the identification of specific genetic factors.

\section{Acknowledgments}

Partial support for all data sets within the UPDB was provided by the Huntsman Cancer Institute, Huntsman Cancer Foundation, University of Utah, and the Huntsman Cancer Institute's Cancer Center Support grant (P30 CA42014) from the National Cancer Institute.

\section{References}

1. Aitken LA, Lindan CE, Sidney S, Gupta N, Barkovich AJ, Sorel M, et al: Chiari type I malformation in a pediatric population. Pediatr Neurol 40:449-454, 2009

2. Atkinson JL, Kokmen E, Miller GM: Evidence of posterior fossa hypoplasia in the familial variant of adult Chiari I malformation: case report. Neurosurgery 42:401-404, 1998

3. Boyles AL, Enterline DS, Hammock PH, Siegel DG, Slifer $\mathrm{SH}$, Mehltretter L, et al: Phenotypic definition of Chiari type I malformation coupled with high-density SNP genome screen shows significant evidence for linkage to regions on chromosomes 9 and 15. Am J Med Genet A 140:2776-2785, 2006

4. Cavender RK, Schmidt JH III: Tonsillar ectopia and Chiari malformations: monozygotic triplets. Case report. J Neurosurg 82:497-500, 1995

5. Coria F, Quintana F, Rebollo M, Combarros O, Berciano J: Occipital dysplasia and Chiari type I deformity in a family. Clinical and radiological study of three generations. J Neurol Sci 62:147-158, 1983

6. George S, Page AB: Familial Arnold-Chiari Type I malformation. Eye (Lond) 20:400-402, 2006

7. Hill J: A survey of cancer sites by kinship in the Utah Mormon population, in Cairns J, Lyon JL, Skolnick M (eds): Banbury Report No. 4. Cancer Incidence in Defined Populations. Cold Spring Harbor, NY: Cold Spring Harbor Laboratory, 1980

8. Malécot G: Les Mathematiques de l'Heredite. Paris: Masson \& Cie, 1948

9. Markunas CA, Enterline DS, Dunlap K, Soldano K, Cope H, Stajich J, et al: Genetic evaluation and application of posterior cranial fossa traits as endophenotypes for Chiari type I malformation. Ann Hum Genet 78:1-12, 2014

10. Markunas CA, Lock E, Soldano K, Cope H, Ding CK, Enterline DS, et al: Identification of Chiari Type I Malformation subtypes using whole genome expression profiles and cranial base morphometrics. BMC Med Genomics 7:39, 2014

11. Markunas CA, Soldano K, Dunlap K, Cope H, Asiimwe E, Stajich J, et al: Stratified whole genome linkage analysis of 
Chiari type I malformation implicates known Klippel-Feil syndrome genes as putative disease candidates. PLoS One 8:e61521, 2013

12. Mavinkurve GG, Sciubba D, Amundson E, Jallo GI: Familial Chiari type I malformation with syringomyelia in two siblings: case report and review of the literature. Childs Nerv Syst 21:955-959, 2005

13. McLellan T, Jorde LB, Skolnick MH: Genetic distances between the Utah Mormons and related populations. Am J Hum Genet 36:836-857, 1984

14. Milhorat TH, Chou MW, Trinidad EM, Kula RW, Mandell M, Wolpert C, et al: Chiari I malformation redefined: clinical and radiographic findings for 364 symptomatic patients. Neurosurgery 44:1005-1017, 1999

15. Miller JH, Limbrick DD, Callen M, Smyth MD: Spontaneous resolution of Chiari malformation Type I in monozygotic twins. J Neurosurg Pediatr 2:317-319, 2008

16. Schanker BD, Walcott BP, Nahed BV, Kahle KT, Li YM, Coumans JV: Familial Chiari malformation: case series. Neurosurg Focus 31(3):E1, 2011

17. Slolnick M: The Utah genealogical database: a resource for genetic epidemiology, in Cairns J, Lyon J, Skolnick M (eds) Banbury Report No. 4. Cancer Incidence in Defined Populations. Cold Spring Harbor, NY: Cold Spring Harbor Laboratory, 1980, pp 285-297

18. Speer MC, George TM, Enterline DS, Franklin A, Wolpert CM, Milhorat TH: A genetic hypothesis for Chiari I malformation with or without syringomyelia. Neurosurg Focus 8(3):E12, 2000
19. Stovner LJ, Cappelen J, Nilsen G, Sjaastad O: The Chiari type I malformation in two monozygotic twins and firstdegree relatives. Ann Neurol 31:220-222, 1992

20. Szewka AJ, Walsh LE, Boaz JC, Carvalho KS, Golomb MR: Chiari in the family: inheritance of the Chiari I malformation. Pediatr Neurol 34:481-485, 2006

\section{Disclosures}

The authors report no conflict of interest concerning the materials or methods used in this study or the findings specified in this paper.

\section{Author Contributions}

Conception and design: Cannon-Albright, Brockmeyer, Neklason. Acquisition of data: Cannon-Albright. Analysis and interpretation of data: Cannon-Albright. Drafting the article: Abbott. Critically revising the article: Cannon-Albright, Brockmeyer, Neklason. Reviewed submitted version of manuscript: all authors. Approved the final version of the manuscript on behalf of all authors:

Cannon-Albright. Statistical analysis: Cannon-Albright, Abbott, Teerlink. Study supervision: Cannon-Albright.

\section{Correspondence}

Lisa Cannon-Albright, 391 Chipeta Way, Ste. D, Salt Lake City, UT 84108-1266. email: lisa.albright@utah.edu. 